\title{
Case Report \\ Biologic Graft Augmentation of Anterior Mesh Erosion Complicated by Poor Wound Healing Secondary to Heavy Tobacco Use
}

\author{
Cheau Williams ${ }^{1,2,3}$, Ethan McBrayer ${ }^{2}$ and Samantha Leggio ${ }^{3, *(D)}$ \\ 1 Colquitt Regional Medical Center, Sterling Center Urology \& Urogynecology, Philadelphia College of \\ Osteopathic Medicine, 115 31st Ave SE, Moultrie, GA 31768, USA; Cwilliams@colquittregional.com or \\ cheau.williams@gmail.com \\ 2 Colquitt Regional Medical Center, Georgia South Family Medicine Residency, Moultrie, GA 31768, USA; \\ ethanscott88@gmail.com \\ 3 Philadelphia College of Osteopathic Medicine, Georgia Campus, Suwanee, GA 30024, USA \\ * Correspondence: samanthaleg@pcom.edu
}

check for updates

Citation: Williams, C.; McBrayer, E.; Leggio, S. Biologic Graft

Augmentation of Anterior Mesh Erosion Complicated by Poor Wound Healing Secondary to Heavy Tobacco Use. Uro 2021, 1, 72-75. https:/ / doi.org/10.3390/uro1030010

Academic Editor: Tommaso Cai

Received: 12 May 2021

Accepted: 25 June 2021

Published: 30 June 2021

Publisher's Note: MDPI stays neutral with regard to jurisdictional claims in published maps and institutional affiliations.

Copyright: (c) 2021 by the authors. Licensee MDPI, Basel, Switzerland. This article is an open access article distributed under the terms and conditions of the Creative Commons Attribution (CC BY) license (https:// creativecommons.org/licenses/by/ $4.0 /)$.

\begin{abstract}
Background: Pelvic organ prolapse (POP) is common among post-menopausal women affecting more than $25 \%$ in their lifetime-with $11 \%$ having a lifetime risk of undergoing an operation for a POP. In April 2019, the Food and Drug Administration (FDA) took surgical mesh for transvaginal use off the market due to safety and effectiveness concerns. This leaves colporrhaphy or colporrhaphy with bio-graft options for a POP surgical repair. (2) Case: In this report, we look at a case with anterior mesh erosion complicated by poor wound healing secondary to heavy tobacco use and how it was successfully removed and augmented with a Coloplast axis allograft dermis biological graft secured with an Anchorsure sacrospinous ligament/arcus tendineus fascia pelvis fixation device and prolene suture. (3) Results: After failing two prior surgeries to rectify the mesh erosion, a final procedure was performed using a biologic dermal graft and a double-layer closure to aid in protecting and increasing the integrity of the tissue. (4) Conclusions: Collectively, the patient and her surgeries highlight the difficult nature of complete mesh removal and how tobacco use can significantly affect the proper healing of surgical sites. The number of surgeries necessary to address the patient's chief complaint and the resolution of her symptoms with the biologic graft supports the challenges one faces with mesh removal and poor wound healing secondary to tobacco use. This case illustrates that complicated transvaginal mesh erosion should initially be augmented with a biologic dermal graft secured via sacrospinous ligament/arcus tendineus fascia pelvis fixation and double-layer closure and not only if visible mesh removal alone fails.
\end{abstract}

Keywords: synthetic mesh with erosion; biologic graft; pelvic organ prolapse; delayed wound healing secondary to tobacco use; poor wound healing; heavy tobacco use

\section{Introduction}

Pelvic organ prolapse (POP) is common among post-menopausal women, affecting more than $25 \%$ in their lifetime. The treatments for POP include the following: Pelvic floor therapy, pessaries, and surgical treatment. The estimated number of women that have a lifetime risk of undergoing an operation for a POP is 11\% [1]. In April 2019, the FDA took surgical mesh for transvaginal use off the market due to safety and effectiveness concerns (mesh erosion). This leaves colporrhaphy or colporrhaphy with bio-graft options for a POP surgical repair. The following will discuss a case with augmentation of an anterior mesh erosion complicated by poor tissue and wound healing with a bio-graft.

\section{Case Report}

DR is a 44-year-old Caucasian woman with a history of prior mesh removal and heavy tobacco use who presented to the office in January 2019 complaining of vaginal 
pain secondary to half a centimeter of mesh exposure at the vaginal apex. The patient also complained of foul-smelling vaginal discharge, bladder spasms, and stress incontinence. The patient was encouraged to quit smoking. In March 2019, she underwent a surgical procedure to remove the exposed mesh from the anterior vaginal wall. The mesh was exposed with a right angle retractor and the area of concern was grasped with 3 Allis clamps for a three-pronged technique. The exposed mesh was then grasped with a Kelly clamp and circumferentially sharply dissected and excised. The defect was closed in a running locking fashion with 2-0 Vicryl suture on a UR 6 needle with good hemostasis. During the 4-week post-op visit, she was doing as expected.

In March 2020, a year after the first mesh removal, she returned with the same complaints of vaginal pain in the anterior wall and foul-smelling vaginal discharge for some time before presenting to the office. Due to the COVID-19 pandemic, all surgeries were postponed. The patient was encouraged to quit smoking and was counseled on the effects smoking has on the body, specifically on tissue healing - the patient claimed to have achieved smoking cessation in March 2020. The patient started on compounded Premarin vaginal cream to improve the health of the vaginal tissue before surgery.

A second surgery took place in July 2020. The exposed vaginal mesh at the left vaginal sulcus area and vaginal apex were excised. Two Allis clamps placed on the area of concern at the vaginal apex allowed for visualization of the granulation tissue and the site of inflammation at the vaginal apex. Electrocautery was used to circumferentially dissect the mesh and granulation tissue from the vaginal apex, inspecting for any other exposed mesh. A UR 6 needle with a 2-0 Vicryl suture was used to close the vaginal epithelium in a running locking fashion with good hemostasis. Six weeks post-op from the second mesh removal, the patient was doing well with some vaginal discharge due to the sutures dissolving. Two months after the second mesh removal, the patient came into the office complaining of vaginal mesh exposure and the defect was closed in the office with 4-0 Monocryl suture in an interrupted fashion $\times 3$. The importance of pelvic rest was emphasized to the patient to allow the vaginal tissue time to heal. The patient continued to experience delayed wound healing secondary to her heavy tobacco use. The patient has previously stated that she stopped smoking in March 2020, but later confessed she "cut down" but did not quit, still leaving her tissue fragile.

During the following visit, the patient continued to complain of vaginal pain secondary to anterior mesh erosion. It was then decided to remove the exposed vaginal mesh and augment the area with a bio-graft to prevent recurrence as she had been dealing with the mesh complications for years and her tissue strength was not improving with the tried measures. Preparing the vaginal mucosa for surgery, she continued to use compounded Premarin vaginal cream nightly for a month.

Figure 1 illustrates that the third and final surgery was in November 2020. The exposed vaginal mesh from her previous procedures in the past was present and was able to be excised from the tissue. After the removal of the frayed polypropylene mesh, a Coloplast axis allograft dermis biologic graft was placed over the friable tissue for protection from any invisible remaining mesh and to help with the integrity of the incision. The graft was then secured using an Anchorsure sacrospinous ligament/arcus tendineus fascia pelvis fixation device and prolene suture. Using 2-0 Vicryl a single layer closure followed by a second layer closure in an interrupted fashion completed the procedure. To help prevent infection Irrisept irrigation was applied to the surgical site allowing for visualization of hemostasis. Five days post-op, the patient was healing well with sutures still in place. Eight weeks post-op, the patient still had some vaginal pain resolved by the 12-week post-op visit. During the 12-week post-op visit there were no signs of anterior mesh, inflammation or infection. By 6 months post-op, she was still doing very well and had no signs of mesh erosion or complaints-she was satisfied with the procedure. 

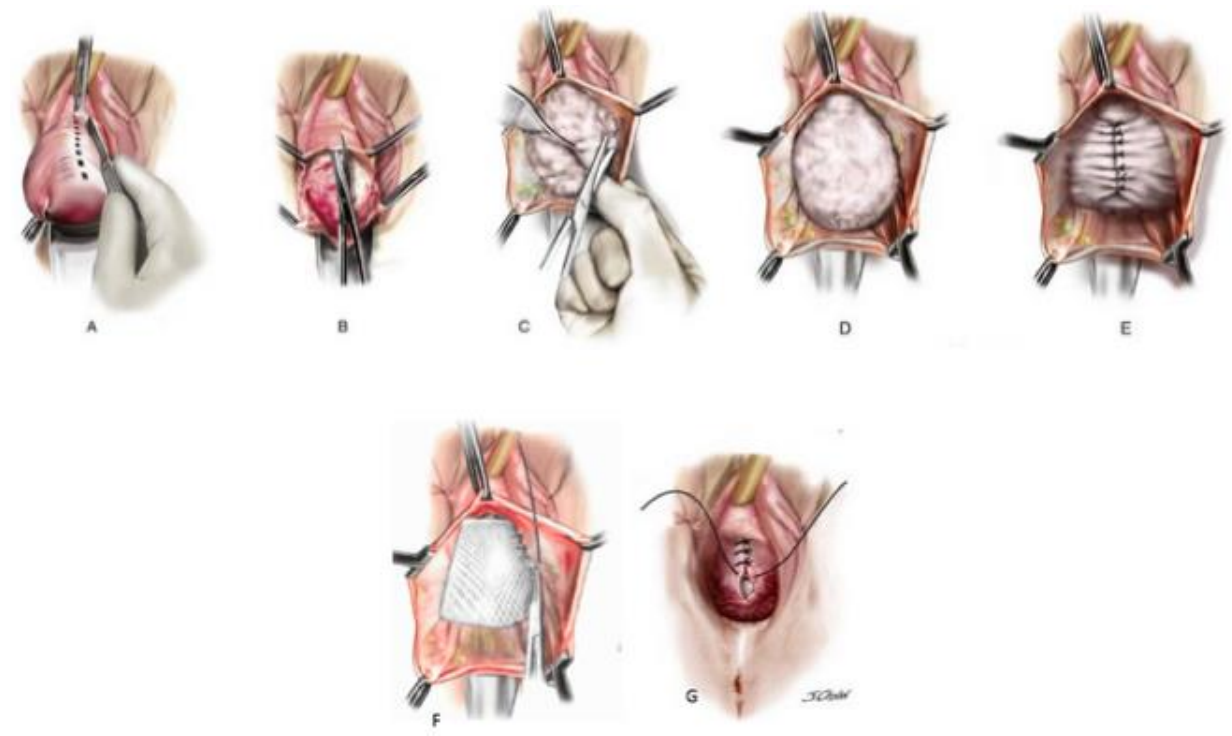

Figure 1. Illustration of a transvaginal anterior wall repair with a biologic graft [2]. (A) Incision into the anterior vaginal wall; (B) Incision into the anterior vaginal wall; (C) Dissection of the pubocervical fascia from the anterior vaginal wall; (D) Visualization of the defect; (E) Plication of the defect; (F) Anchorsure deployment bilaterally into the sacrospinous ligaments and arcus tendineus fascia pelvis, the prolene suture is pierced through the dermal bio-graft and tied down creating a lift and barrier between the bladder and anterior vaginal wall *; (G) Trimming and re-approximation of the vaginal tissue. * A demonstration and visualization of the Anchorsure is available at the following link: https: / / www.youtube.com/watch?v=9oBXxdRu5gk [3] (accessed on 23 October 2017).

\section{Discussion}

The estimated number of women who have a lifetime risk of undergoing a single operation for a POP is $11 \%$. Risk factors for POP consist of older age, post-menopausal status, multiparty, and obesity. The demand for services to treat pelvic floor disorders is expected to increase by $45 \%$ by the year 2030, leading to an increase in demand for effective and safe surgical options. Based on the Gomelsky et al. paper, any graft significantly reduces objective prolapse recurrence rates compared to no graft. Gomelsky et al. concluded that there is a lack of data supporting the use of biologics in pelvic prolapse repair and that more randomized controlled trials must be conducted to determine which graft is superior-synthetic vs. biologic [1].

Both biologic and synthetic grafts have their own set of complications. In a study done by Sohlberg et al., biologic grafts led to an increased surgery rate for recurrence, and synthetic rates led to repeat surgeries for graft complications [4]. The meta-analysis done by Slade et al. noted, as well as other studies, that the long-term safety of mesh was unclear and that more studies needed to be conducted [5]. Due to such complications with synthetic mesh, the FDA did pull synthetic mesh off the market in April 2019 due to safety and effectiveness concerns.

Gomelsky et al. noted how more clinical trials were needed to determine the effectiveness and safety of bio-grafts. McAlvany et al. did so and determined that dermal bio-grafts for POP repair were an effective and safe procedure [6]. Juma et al. conducted a longitudinal study looking at solvent-dehydrated dermal allograft augmented cystocele repair and concluded that augmented anterior repair is a safe procedure, with low morbidity, and its success is comparable to other techniques [7].

Based on the research available, the synthetic mesh has an increased risk of postoperative complications, such as mesh erosion among others, and colporrhaphy alone has an increased risk of POP recurrence leaving colporrhaphy augmented with a dermal bio-graft as the safe and effective option for surgical POP repair, as well as augmentation for mesh erosion, especially in patients with poor tissue and poor wound healing. 


\section{Conclusions}

Collectively, the patient and her surgeries highlight the difficult nature of complete mesh removal and how tobacco use can significantly affect the proper healing of surgical sites. The number of surgeries necessary to address the patient's chief complaint and the resolution of her symptoms with the biologic graft supports the challenges one faces with mesh removal and poor wound healing secondary to tobacco use. We hope to aid in the advancement of biological alternatives for the augmentation of mesh erosions in POP preventing the necessity of multiple surgical corrections. This case illustrates that complicated transvaginal mesh erosion should initially be augmented with a biologic dermal graft secured via sacrospinous ligament/arcus tendineus fascia pelvis fixation and double-layer closure and not only if visible mesh removal alone fails.

Author Contributions: S.L. collected the data and typed the article; E.M. collected the references and edited the article; C.W. examined, operated on the patient, and edited the article. All authors have read and agreed to the published version of the manuscript.

Funding: No funding was received for this article.

Institutional Review Board Statement: Ethical review and approval were waived for this study, due to the nature of the case report being an observatory of the standard of care for the patient's diagnosis and not experimental.

Informed Consent Statement: Informed consent was obtained from the patient.

Conflicts of Interest: Williams is a surgical consultant of Coloplast Urology.

\section{References}

1. Gomelsky, A.; Dmochowski, R.R. Biologic Material for Pelvic Floor Reconstruction. Curr. Bladder. Dysfunct Rep. 2012, 7, 201-209. [CrossRef]

2. Themes, U.F.O. Surgical Management of Anterior Vaginal Wall Prolapse. Abdominal Key. Available online: https: / /abdominalkey. $\mathrm{com} /$ surgical-management-of-anterior-vaginal-wall-prolapse/ (accessed on 24 July 2016).

3. NEOMEDIC Incorporated. ANCHORSURE Virtual Surgery. YouTube. Available online: https://www.youtube.com/watch?v= 9oBXxdRu5gk (accessed on 23 October 2017).

4. Sohlberg, E.M.; Dallas, K.B.; Weeks, B.T.; Elliott, C.S.; Rogo-Gupta, L. Reoperation rates for pelvic organ prolapse repairs with biologic and synthetic grafts in a large population-based cohort. Int. Urogynecol. J. 2020, 31, 291-301. [CrossRef]

5. Slade, E.; Daly, C. Primary Surgical Management of Anterior Pelvic Organ Prolapse: A Systematic Review, Network Metaanalysis, and Cost-Effectiveness Analysis. BJOG Int. J. Obstet. Gynaecol. 2019. Available online: https:/ /www.researchgate.net/ publication/335756490_Primary_surgical_management_of_anterior_pelvic_organ_prolapse_a_systematic_review_network_ meta-analysis_and_cost-effectiveness_analysis (accessed on 12 November 2020).

6. McAlvany, K.; Piraino, J.; Bendana, E.; Secrest, C. V2-04 Allograft Dermis for Female Pelvic Floor Repair. Available online: https:/ / www.auajournals.org/doi/full/10.1016/j.juro.2016.02.2658 (accessed on 12 November 2020).

7. Juma, S.; Raheem, O.A. Solvent-dehydrated dermal allograft (AXISTM) augmented cystocele repair: Longitudinal results. Int. Urogynecol. J. 2017, 28, 1159-1164. [CrossRef] 